\title{
Suicide Inactivation of Dioldehydratase by Glycolaldehyde and Chloroacetaldehyde: An Examination of the Reaction Mechanism
}

\author{
Gregory M. Sandala, ${ }^{\dagger, \pi}$ David M. Smith, ${ }^{\ddagger,}{ }^{\star}$ Michelle L. Coote ${ }^{\dagger}$ and Leo Radom ${ }^{\dagger, \pi, *}$ \\ School of Chemistry, University of Sydney, Sydney, NSW 2006, Australia, Research School of \\ Chemistry, Australian National University, Canberra, ACT 0200, Australia, and Rudjer Boskovic \\ Institute, Zagreb, Croatia
}

\section{SUPPORTING INFORMATION}

(Tables S1-S2, Total 10 pages including this page)

$\dagger$ Australian National University

I University of Sydney

\$ Rudjer Boskovic Institute, Zagreb, Croatia

*E-mail: david.smith@irb.hr; radom@chem.usyd.edu.au 


\section{Details of Calculations}

Calculations were performed with Revisions B.03 and B.05 of GAUSSIAN 03, ${ }^{1}$ and with the MOLPRO $2000.6^{2}$ computational packages. Calculations were performed at a high level of theory, chosen on the basis of a recent assessment study. ${ }^{3}$ Geometries and scaled zero-point vibrational energies were obtained at the UMPW $1 \mathrm{~K} / 6-31+\mathrm{G}(\mathrm{d}, \mathrm{p})$ level of theory. ${ }^{4}$ Improved relative energies were obtained with an improved version $^{5}$ of the originally reported ${ }^{6}$ G3(MP2)-RAD methodology.

(1) Frisch, M. J.; Trucks, G. W.; Schlegel, H. B.; Scuseria, G. E.; Robb, M. A.; Cheeseman, J. R.; Montgomery, J. A., Jr.; T. V.; Kudin, K. N.; Burant, J. C.; Millam, J. M.; Iyengar, S. S.; Tomasi, J.; Barone, V.; Mennucci, B.; Cossi, M.; Scalmani, G.; Rega, N.; Petersson, G. A.; Nakatsuji, H.; Hada, M.; Ehara, M.; Toyota, K.; Fukuda, R.; Hasegawa, J.; Ishida, M.; Nakajima, T.; Honda, Y.; Kitao, O.; Nakai, H.; Klene, M.; Li, X.; Knox, J. E.; Hratchian, H. P.; Cross, J. B.; Adamo, C.; Jaramillo, J.; Gomperts, R.; Stratmann, R. E.; Yazyev, O.; Austin, A. J.; Cammi, R.; Pomelli, C.; Ochterski, J. W.; Ayala, P. Y.; Morokuma, K.; Voth, G. A.; Salvador, P.; Dannenberg, J. J.; Zakrzewski, G.; Dapprich, S.; Daniels, A. D.; Strain, M. C.; Farkas, O.; Malick, D. K.; Rabuck, A. D.; Raghavachari, K.; Foresman, J. B.; Ortiz, J. V.; Cui, Q.; Baboul, A. G.; Clifford, S.; Cioslowski, J.; Stefanov, B. B.; Liu, G.; Liashenko, A.; Piskorz, P.; Komaromi, I.; Martin, R. L.; Fox, D. J.; Keith, T.; Al-Laham, M. A.; Peng, C. Y.; Nanayakkara, A.; Challacombe, M.; Gill, P. M. W.; Johnson, B.; Chen, W.; Wong, M. W.; Gonzalez, C.; Pople, J. A. Gaussian 03; Gaussian, Inc.: Pittsburgh, PA, 2003.

(2) Werner, H.-J.; Knowles, P. J.; Amos, R. D.; Bernhardsson, A.; Berning, A.; Celani, P.; Cooper, D. L.; Deegan, M. J. O.; Dobbyn, A. J.; Eckert, F.; Hampel, C.; Hetzer, G.; Korona, T.; Lindh, R.; Lloyd, A. W.; McNicholas, S. J.; Manby, F. R.; Meyer, W.; Mura, M. E.; Nicklass, A.; Palmieri, P.; Pitzer, R.; Rauhut, G.; Schütz, M.; Stoll, H.; Stone, A. J.; Tarroni, R.; Thorsteinsson, T. MOLPRO 2000.6; University of Birmingham: Birmingham, 1999.

(3) Coote, M. L. J. Phys. Chem. A. 2004, 108, 3865-3872.

(4) (a) Lynch, B. J.; Fast, P. L.; Harris, M.; Truhlar, D. G. J. Phys. Chem. A. 2000, 104, 4811-4815. (b) Lynch, B. J.; Truhlar, D. G. J. Phys. Chem. A. 2001, 105, 2936-2941.

(5) Henry, D. J.; Sullivan, M. B.; Radom, L. J. Chem. Phys. 2003, 118 4849-4860.

(6) Henry, D. J.; Parkinson, C. J.; Mayer, P. M.; Radom, L. J. Phys. Chem. A. 2001, 105, 6750-6756. 


\section{TABLE S1: GAUSSIAN Archive Entries for the MPW1K/6-31+G(d,p) Optimized Geometries}

1

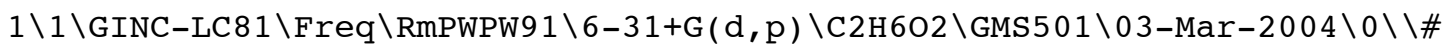
P MPWPW91/6-31+G(D,P) FREQ=NORAMAN GFINPUT MAXDISK $=30146560$ IOP $(3 / 76=0$ $572004280) \backslash \backslash 1,2$-diolethane freq iop $=1 \operatorname{MPW} 1 \mathrm{~K} / 6-31+\mathrm{G}(\mathrm{d}, \mathrm{p}) / / \backslash 0,1 \backslash \mathrm{C}, 0.680$ $7859706,0.5985368529,-0.2612600882 \backslash \mathrm{C},-0.7303520294,0.5642718529,0.2651$ $739118 \backslash 0,1.3132449706,-0.5697121471,0.2126259118 \backslash 0,-1.4182070294,-0.56$ $62061471,-0.1893780882 \backslash \mathrm{H},-0.7051660294,0.5946438529,1.3582659118 \backslash \mathrm{H},-1$. $2772120294,1.4369908529,-0.0845760882 \backslash \mathrm{H}, 0.6608639706,0.6124568529,-1.3$ $529620882 \backslash \mathrm{H}, 1.1919019706,1.4961838529,0.0958999118 \backslash \mathrm{H},-0.8748120294,-1$. $3269981471,0.0130889118 \backslash \mathrm{H}, 2.1415169706,-0.7027831471,-0.2391820882 \backslash \backslash \mathrm{Ve}$ rsion $=x 86-$ Linux-G03RevB. 03 \State $=1-A \backslash H F=-230.1993219 \backslash$ RMSD $=4.051 e-09 \backslash R M$ $\mathrm{SF}=2.381 \mathrm{e}-06 \backslash \mathrm{Dipole}=0.9731369,0.4176031,-0.1980609 \backslash \mathrm{PG}=\mathrm{C} 01[\mathrm{X}(\mathrm{C} 2 \mathrm{H} 6 \mathrm{O} 2)] \backslash \backslash \mathrm{Q}$

\section{2}

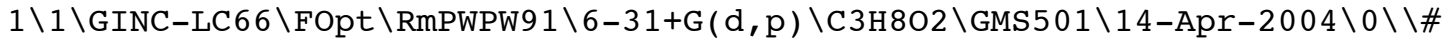
P MPWPW91/6-31+G (D, P ) OPT $=($ TIGHT, MAXCYCLE $=100)$ MAXDISK=36044800 GFINPU

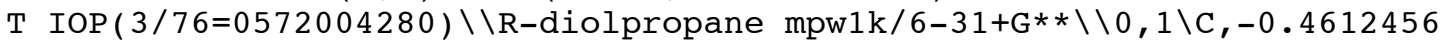
$773,0.0583485559,-0.3375192368 \backslash \mathrm{C}, 0.7079035803,-0.7340710086,0.20249633$ $58 \backslash 0,1.8758045061,-0.027049197,-0.1515370959 \backslash \mathrm{H}, 0.6146155682,-0.8124503$ $133,1.2884301232 \backslash \mathrm{H}, 0.7120195106,-1.7383812311,-0.2277178932 \backslash \mathrm{C},-1.78611$ $17491,-0.5612021572,0.0304658439 \backslash 0,-0.4311111837,1.3632517488,0.180635$ $15 \backslash \mathrm{H},-0.363445019,0.0931157099,-1.4284280432 \backslash \mathrm{H},-1.8957111966,-0.611648$ $636,1.1122983264 \backslash \mathrm{H},-2.598550059,0.0444468152,-0.3619275368 \backslash \mathrm{H},-1.876282$ $5914,-1.565131057,-0.3803889373 \backslash \mathrm{H}, 2.6256091672,-0.3693074957,0.3264542$ $451 \backslash \mathrm{H}, 0.4609211177,1.6912834524,0.0658376257 \backslash \backslash$ Version=x86-Linux-G03Rev B. 05 $\backslash$ State $=1-A \backslash H F=-269.514911 \backslash R M S D=9.089 e-09 \backslash R M S F=1.633 e-06 \backslash D i p o l e=0.5$ $363873,-0.8821293,0.1881809 \backslash \mathrm{PG}=\mathrm{C} 01[\mathrm{X}(\mathrm{C} 3 \mathrm{H} 8 \mathrm{O} 2)] \backslash \backslash \mathrm{Q}$

\section{3}

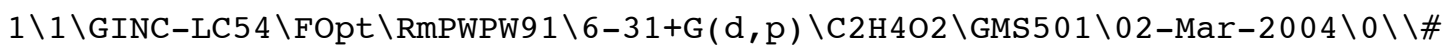
P MPWPW91/6-31+G(D,P) OPT=TIGHT MAXDISK $=32768000$ IOP $(3 / 76=0572004280) \backslash$ $\backslash$ Glycolaldehyde iop=1 MPW $1 \mathrm{~K} / 6-31+\mathrm{G}(\mathrm{d}, \mathrm{p}) / /$ opt $\backslash \backslash 0,1 \backslash \mathrm{H}, 1.3535158324,-0.4$ $142317368,0 . \backslash 0,1.3303866561,0.5467586502,0$. \C, $0.0006193041,0.926142632$ $8,0 . \backslash \mathrm{H},-0.2362810259,1.5374495658,0.8776076985 \backslash \mathrm{H},-0.2362810259,1.53744$ $95658,-0.8776076985 \backslash \mathrm{C},-0.9202256253,-0.2526791417,0 . \backslash 0,-0.5008363799,-$ $1.3790970169,0 . \backslash \mathrm{H},-1.9997180634,-0.0427414082,0 . \backslash \backslash$ Version=x86-Linux-G0 3RevB. 03 \State $=1-A^{\prime} \backslash H F=-228.9772435 \backslash R M S D=2.464 e-09 \backslash R M S F=3.337 e-06 \backslash D i p o$ $1 \mathrm{e}=-0.8732797,0.6177588,0 . \backslash \mathrm{PG}=\mathrm{CS} \quad[\mathrm{SG}(\mathrm{C} 2 \mathrm{H} 2 \mathrm{O} 2), \mathrm{X}(\mathrm{H} 2)] \backslash \backslash \mathrm{a}$

\section{4}

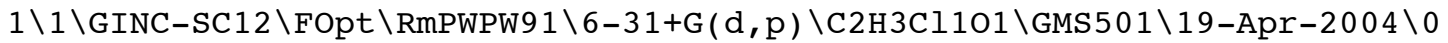
$\backslash \backslash \# P$ MPWPW91/6-31+G(D,P) OPT=(TIGHT, MAXCYCLE $=100)$ MAXDISK=32768000 GFI

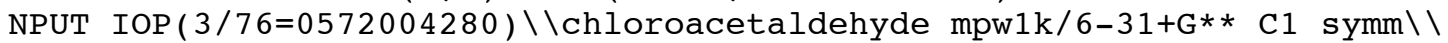
$0,1 \backslash 0,2.2890015128,-0.0168049766,-0.0816404743 \backslash \mathrm{C}, 1.1547108077,-0.35655$ $62924,0.0874765687 \backslash \mathrm{C}, 0.0221253962,0.6383112229,0.043829465 \backslash \mathrm{Cl},-1.55578$ $21318,-0.1581365254,-0.0325991192 \backslash \mathrm{H}, 0.1390016133,1.2846152973,-0.81991$ $24692 \backslash \mathrm{H}, 0.0555504594,1.2493933189,0.9423652032 \backslash \mathrm{H}, 0.8807148426,-1.40177$ $74542,0.2970198854 \backslash \backslash$ Version=DEC-AXP-OSF $/ 1-$ G03RevB .05 State $=1-A \backslash H F=-613$ $.3848357 \backslash \mathrm{RMSD}=5.557 \mathrm{e}-09 \backslash \mathrm{RMSF}=1.880 \mathrm{e}-06 \backslash \mathrm{Dipole}=-0.3766128,0.1770302,0.1$ $79351 \backslash \mathrm{PG}=\mathrm{C} 01 \quad[\mathrm{X}(\mathrm{C} 2 \mathrm{H} 3 \mathrm{C} 1101)] \backslash \backslash \mathrm{Q}$ 


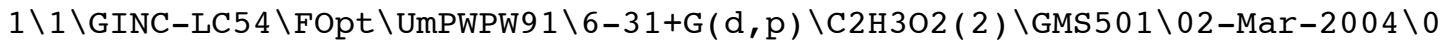
$\backslash \backslash \# \mathrm{P}$ MPWPW91/6-31+G(D,P) OPT=(TIGHT, MAXCYCLE $=100)$ MAXDISK=26214400 GFI NPUT IOP $(3 / 76=0572004280) \backslash \backslash$ Semidione ethane $c 2 \mathrm{v}$ MPW $1 \mathrm{~K} / 6-31+\mathrm{G}(\mathrm{d}, \mathrm{p}) / /$ io $\mathrm{p}=1 \backslash \backslash 0,2 \backslash \mathrm{H}, 0 ., 0 ., 1.0237672834 \backslash 0,0.1 .1480866315,0.5795396785 \backslash 0,0 .,-1.1$ $480866315,0.5795396785 \backslash \mathrm{C}, 0 ., 0.7083308496,-0.6134019275 \backslash \mathrm{C}, 0 \ldots,-0.7083308$ $496,-0.6134019275 \backslash \mathrm{H}, 0 ., 1.3678952885,-1.4677895048 \backslash \mathrm{H}, 0 .,-1.3678952885,-$ $1.4677895048 \backslash \backslash$ Version $=x 86-L$ inux-G03RevB.03 $\backslash$ State $=2-B 1 \backslash H F=-228.3275025 \backslash$ $\mathrm{S} 2=0.756604 \backslash \mathrm{S} 2-1=0 . \backslash \mathrm{S} 2 \mathrm{~A}=0.750025 \backslash \mathrm{RMSD}=5.308 \mathrm{e}-09 \backslash \mathrm{RMSF}=2.282 \mathrm{e}-06 \backslash \mathrm{Dipole}=$ $0 ., 0 .,-1.1440872 \backslash \mathrm{PG}=\mathrm{C} 02 \mathrm{~V} \quad[\mathrm{C} 2(\mathrm{H} 1), \mathrm{SGV}(\mathrm{C} 2 \mathrm{H} 2 \mathrm{O} 2)] \backslash \backslash @$

\section{6}

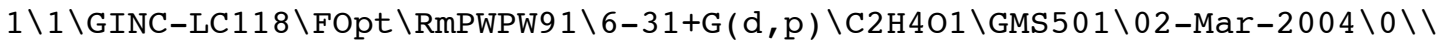
\#P MPWPW $1 / 6-31+G(D, P) \quad O P T=(T I G H T, M A X C Y C L E=100) \quad M A X D I S K=32768000$ IOP $(3$ $/ 76=0572004280) \backslash \backslash$ acetaldehyde iop $=1 \mathrm{MPW} 1 \mathrm{~K} / 6-31+\mathrm{G}(\mathrm{d}, \mathrm{p}) / /$ opt $\backslash \backslash 0,1 \backslash \mathrm{C}, 0.9$ $246700951,-0.7138747453,0 . \backslash \mathrm{C}, 0.0023866972,0.4592052866,0 . \backslash 0,-1.1954287$ $198,0.3823437254,0 . \backslash \mathrm{H}, 0.4907996257,1.4492193882,0 . \backslash \mathrm{H}, 1.5730160079,-0.6$ $685315027,0.8750890374 \backslash \mathrm{H}, 1.5730160079,-0.6685315027,-0.8750890374 \backslash \mathrm{H}, 0$. $364257363,-1.6428894332,0 . \backslash$ Version $=x 86-$ Linux-G03RevB.03 $\backslash$ State $=1-A^{\prime} \backslash$ HF $=-153.7884354 \backslash \mathrm{RMSD}=1.552 \mathrm{e}-09 \backslash \mathrm{RMSF}=1.353 \mathrm{e}-06 \backslash \mathrm{Dipole}=1.199514,-0.1148504$ , $0 . \backslash P G=C S[S G(C 2 \mathrm{H} 2 \mathrm{O} 1), X(\mathrm{H} 2)] \backslash \backslash @$

\section{7}

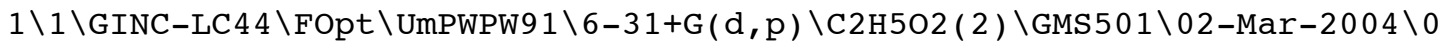
$\backslash \backslash \# P$ MPWPW91/6-31+G(D,P) OPT=TIGHT GFINPUT MAXDISK $=32768000$ IOP $(3 / 76=0$ $572004280) \backslash \backslash 1,2$-diolethane radical $\mathrm{MPW} 1 \mathrm{~K} / 6-31+\mathrm{G}(\mathrm{d}, \mathrm{p}) / /$ opt iop $=1 \backslash \backslash 0,2 \backslash \mathrm{C}$ $, 0.7335321735,0.5675684198,-0.2575224613 \backslash \mathrm{C},-0.6800243895,0.6306270225$, $0.1847291177 \backslash \mathrm{O}, 1.4423032407,-0.5143305497,0.1281028807 \backslash \mathrm{H}, 0.8210463182$, $-1.2268172986,0.2953653176 \backslash \mathrm{H}, 1.3321561123,1.4538067748,-0.3933754667 \backslash \mathrm{O}$ $,-1.3306422434,-0.6021711705,-0.0575073745 \backslash \mathrm{H},-1.4147247023,-0.72267742$ $43,-1.0019988849 \backslash \mathrm{H},-0.7603380465,0.7726817752,1.2667300875 \backslash \mathrm{H},-1.192474$ $3638,1.4658472798,-0.2947250424 \backslash \backslash$ Version=x86-Linux-G03RevB.03\State=2$\mathrm{A} \backslash \mathrm{HF}=-229.5401787 \backslash \mathrm{S} 2=0.754976 \backslash \mathrm{S} 2-1=0 . \backslash \mathrm{S} 2 \mathrm{~A}=0.750018 \backslash \mathrm{RMSD}=4.265 \mathrm{e}-09 \backslash \mathrm{RMSF}$ $=2.925 e-06 \backslash \mathrm{Dipole}=-0.3972524,0.3685314,-0.3197755 \backslash \mathrm{PG}=\mathrm{C} 01[\mathrm{X}(\mathrm{C} 2 \mathrm{H} 5 \mathrm{O} 2)] \backslash \backslash @$

\section{8}

$1 \backslash 1 \backslash$ GINC-LC1 $40 \backslash$ FOpt \UmPWPW9 $1 \backslash 6-31+\mathrm{G}(\mathrm{d}, \mathrm{p}) \backslash \mathrm{C} 2 \mathrm{H} 5 \mathrm{O} 2(2) \backslash \mathrm{GMS} 501 \backslash 02-M a r-2004 \backslash$ $0 \backslash \backslash \# \mathrm{P}$ MPWPW91/6-31+G(D,P) OPT=TIGHT GFINPUT MAXDISK $=32768000$ IOP $(3 / 76=$ $0572004280) \backslash \backslash$ gem-diolethane radical iop $=1 \mathrm{MPW} 1 \mathrm{~K} / 6-31+\mathrm{G}(\mathrm{d}, \mathrm{p}) / /$ opt $\backslash \backslash 0,2$ $\backslash \mathrm{C}, 1.402944921,-0.1985726299,-0.0896934192 \backslash \mathrm{H}, 2.1372683074,0.5507655361$ $, 0.1559577245 \backslash \mathrm{H}, 1.697227689,-1.1167741845,-0.5695714998 \backslash \mathrm{C}, 0.0007375106$ $,-0.0052291515,0.3468050384 \backslash 0,-0.7502344603,-1.0610615742,-0.167620733$ $7 \backslash \mathrm{H},-0.0715377082,0.0190116797,1.4386473073 \backslash \mathrm{O},-0.518318851,1.234708996$ $5,-0.0445722837 \backslash \mathrm{H},-0.3810853405,1.3304865423,-0.9870182912 \backslash \mathrm{H},-1.655541$ $0475,-0.9498582641,0.1168591831 \backslash \backslash$ Version $=x 86-$ Linux-G03RevB.03 \State=2$\mathrm{A} \backslash \mathrm{HF}=-229.5430536 \backslash \mathrm{S} 2=0.755316 \backslash \mathrm{S} 2-1=0 . \backslash \mathrm{S} 2 \mathrm{~A}=0.750017 \backslash \mathrm{RMSD}=9.678 \mathrm{e}-09 \backslash \mathrm{RMSF}$ $=7.481 \mathrm{e}-07 \backslash \mathrm{Dipole}=0.0730198,0.0152236,-0.0197735 \backslash \mathrm{PG}=\mathrm{C} 01[\mathrm{X}(\mathrm{C} 2 \mathrm{H} 5 \mathrm{O} 2)] \backslash \backslash @$ 
$1 \backslash 1 \backslash$ GINC-LC5 $4 \backslash$ FOpt $\backslash$ RmPWPW9 $1 \backslash 6-31+G(\mathrm{~d}, \mathrm{p}) \backslash \mathrm{C} 2 \mathrm{H} 6 \mathrm{O} 2 \backslash \mathrm{GMS} 501 \backslash 02-M a r-2004 \backslash 0 \backslash \backslash \#$ P MPWPW91/6-31+G(D,P) OPT $=($ TIGHT, MAXCYCLE $=100)$ MAXDISK $=32768000$ IOP (3/ $76=0572004280) \backslash \backslash$ gem-diolethane1 iop=1 MPW1K/6-31+G(d,p)//opt $\backslash \backslash 0,1 \backslash \mathrm{C}, 1$. $3931516553,-0.0106945554,-0.0849642336 \backslash \mathrm{C},-0.0460974884,-0.0001552432,0$ $.3584183061 \backslash \mathrm{O},-0.6478304009,-1.1467375586,-0.162335833 \backslash \mathrm{H},-1.5615672898$ $,-1.159920968,0.117052845 \backslash 0,-0.7270859133,1.1507402432,-0.0395271804 \backslash \mathrm{H}$ $,-0.6525196915,1.2323050707,-0.9898798275 \backslash \mathrm{H},-0.1165157372,0.0089670315$ $, 1.4471563574 \backslash \mathrm{H}, 1.8968950947,-0.8996158295,0.284606718 \backslash \mathrm{H}, 1.4462859578$, $-0.0207600978,-1.1730540517 \backslash \mathrm{H}, 1.9044271781,0.8721021083,0.2882976312 \backslash \backslash$ Version $=x 86-L$ inux-G03RevB.03 $\backslash$ State $=1-A \backslash H F=-230.2170192 \backslash \mathrm{RMSD}=2.879 \mathrm{e}-09 \backslash$ $\mathrm{RMSF}=1.777 \mathrm{e}-06 \backslash \mathrm{Dipole}=0.0700299,0.0156229,-0.0528971 \backslash \mathrm{PG}=\mathrm{C} 01 \quad[\mathrm{X}(\mathrm{C} 2 \mathrm{H} 6 \mathrm{O} 2)$ ]$\backslash \backslash @$

\section{0}

$1 \backslash 1 \backslash$ GINC-LC1 $40 \backslash$ FOpt \RmPWPW91 $\backslash 6-31+\mathrm{G}(\mathrm{d}, \mathrm{p}) \backslash \mathrm{C} 2 \mathrm{H} 6 \mathrm{O} \backslash \mathrm{GMS} 501 \backslash 02-\mathrm{Mar}-2004 \backslash 0 \backslash \backslash$ \#P MPWPW91/6-31+G(D,P) OPT=TIGHT MAXDISK=39321600 IOP $(3 / 76=0572004280)$

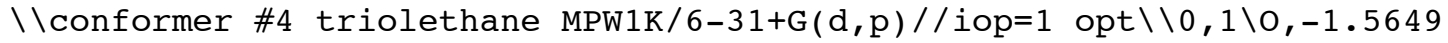
$348298,0.0271747225,-0.5546018268 \backslash \mathrm{C},-0.9068095909,-0.0635628795,0.6821$ $205887 \backslash \mathrm{C}, 0.5877030238,0.0310173185,0.4676492022 \backslash \mathrm{H},-1.2430172483,0.7767$ $449252,1.2825238374 \backslash \mathrm{H},-1.1485448573,-0.9909635429,1.2024987492 \backslash \mathrm{O}, 0.926$ $4121776,1.1852197099,-0.218296093 \backslash 0,0.9392036818,-1.1152140655,-0.2589$ $521432 \backslash \mathrm{H}, 1.1248325431,0.0662621219,1.4160644373 \backslash \mathrm{H},-1.3209405844,-0.740$ $8322245,-1.0703209979 \backslash \mathrm{H}, 0.2623205926,1.3134738285,-0.8999678208 \backslash \mathrm{H}, 1.83$ $45407199,-1.0068546781,-0.5746164466 \backslash \backslash$ Version=x86-Linux-G03RevB.03\Sta te $=1-\mathrm{A} \backslash \mathrm{HF}=-305.4091103 \backslash \mathrm{RMSD}=4.274 \mathrm{e}-09 \backslash \mathrm{RMSF}=3.550 \mathrm{e}-06 \backslash \mathrm{Dipole}=0.2488835$, $-0.3882108,0.1343673 \backslash P G=\mathrm{C} 01 \quad[\mathrm{X}(\mathrm{C} 2 \mathrm{H} 6 \mathrm{O} 3)] \backslash \backslash @$

\section{1}

$1 \backslash 1 \backslash$ GINC-LC1 $12 \backslash$ FOpt $\backslash$ RmPWPW9 $1 \backslash 6-31+G(d, p) \backslash C 2 H 5 C 1102 \backslash G M S 501 \backslash 03-M a y-2004 \backslash$ $0 \backslash \backslash \# P$ MPWPW91/6-31+G (D,P) OPT $=($ TIGHT, MAXCYCLE $=100) \quad$ MAXDISK $=39321600 \mathrm{GF}$ INPUT IOP(3/76=0572004280)\\lowest conformer gemdiol chloroacetaldehyd e mpw $1 \mathrm{k} / 6-31+\mathrm{G} * * \mathrm{C} 1$ symm $\backslash \backslash 0,1 \backslash \mathrm{Cl}, 1.608483974,0.0206606349,-0.300717601$ $9 \backslash \mathrm{C}, 0.3598460552,-0.3037995467,0.9247245046 \backslash \mathrm{C},-1.0298352209,-0.0271905$ $824,0.3926735843 \backslash \mathrm{O},-1.1801131402,1.2570100314,-0.0991487736 \backslash \mathrm{H}, 0.447918$ $705,-1.3439218257,1.2166548763 \backslash \mathrm{H}, 0.5648185499,0.3438802823,1.769472733$ $5 \backslash \mathrm{H},-0.4960905462,1.4094271616,-0.7546506586 \backslash \mathrm{O},-1.3140082755,-0.993013$ $0827,-0.5643623141 \backslash \mathrm{H},-1.7233131866,-0.0952901282,1.2345958697 \backslash \mathrm{H},-2.164$ $6547602,-0.7913610983,-0.9501734214 \backslash \backslash$ Version=x86-Linux-G03RevB.05 $\backslash$ Stat $\mathrm{e}=1-\mathrm{A} \backslash \mathrm{HF}=-689.8174239 \backslash \mathrm{RMSD}=4.968 \mathrm{e}-09 \backslash \mathrm{RMSF}=1.058 \mathrm{e}-06 \backslash \mathrm{Dipole}=-0.4801073$, $-0.0850933,0.4468997 \backslash \mathrm{PG}=\mathrm{C} 01 \quad[\mathrm{X}(\mathrm{C} 2 \mathrm{H} 5 \mathrm{Cl} 1 \mathrm{O} 2)] \backslash \backslash @$ 


\section{2}

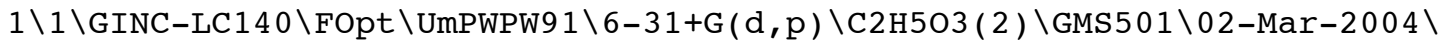
$0 \backslash \backslash \# \mathrm{P}$ MPWPW91/6-31+G(D,P) OPT=TIGHT MAXDISK=26214400 IOP(3/76=05720042 $80) \backslash \backslash$ trioleth_radical iop=1 MPW $1 \mathrm{~K} / 6-31+\mathrm{G}(\mathrm{d}, \mathrm{p}) / \mathrm{opt} / \backslash \backslash 0,2 \backslash 0,1.8925801087$ $,-0.079076114,-0.0570572365 \backslash \mathrm{C}, 0.732880019,-0.7551369253,-0.026925785 \backslash \mathrm{C}$ $,-0.5031157197,-0.0179416648,0.357837059 \backslash \mathrm{H}, 0.830491199,-1.8014260345,0$ $.2126279011 \backslash 0,-1.6457572458,-0.6340308167,-0.1267986053 \backslash 0,-0.334740909$ $3,1.2831734716,-0.1386949282 \backslash \mathrm{H},-0.6496501075,0.0213391983,1.442398582 \backslash$ $\mathrm{H}, 1.6845869674,0.8481555118,-0.199283086 \backslash \mathrm{H},-1.505860347,-0.8316272672$, $-1.053558475 \backslash \mathrm{H},-1.0348091359,1.8414978048,0.1927535934 \backslash \backslash$ Version=x86-Li nux-G03RevB.03 \State $=2-\mathrm{A} \backslash \mathrm{HF}=-304.7473918 \backslash \mathrm{S} 2=0.75491 \backslash \mathrm{S} 2-1=0 . \backslash \mathrm{S} 2 \mathrm{~A}=0.7500$ $17 \backslash \mathrm{RMSD}=9.067 \mathrm{e}-09 \backslash \mathrm{RMSF}=3.045 \mathrm{e}-06 \backslash \mathrm{Dipole}=-0.3826998,0.4322094,0.1264291$ $\backslash \mathrm{PG}=\mathrm{C} 01 \quad[\mathrm{X}(\mathrm{C} 2 \mathrm{H} 5 \mathrm{O} 3)] \backslash \backslash @$

\section{3}

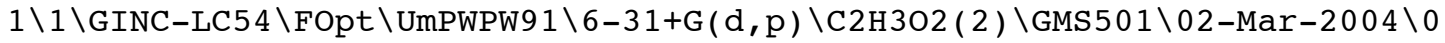
$\backslash \backslash \# P$ MPWPW91/6-31+G(D,P) OPT= (MAXCYCLE $=100$, TIGHT $)$ MAXDISK=26214400 IOP $(3 / 76=0572004280) \backslash \backslash \mathrm{glycolrad} i o p=1 \mathrm{MPW} 1 \mathrm{~K} / 6-31+\mathrm{G}(\mathrm{d}, \mathrm{p}) / /$ opt $\backslash \backslash 0,2 \backslash \mathrm{H}, 1.35$ $17034796,-0.3670811153,0 . \backslash 0,1.2927328858,0.6005095921,0 . \backslash \mathrm{C}, 0.009188400$ $6,0.889670784,0 . \backslash \mathrm{C},-0.9177774955,-0.1761607767,0 . \backslash 0,-0.5023328009,-1.3$ $389987407,0 . \backslash \mathrm{H},-0.2381366432,1.9376305652,0 . \backslash \mathrm{H},-1.9852329461,0.0563036$ $949,0 . \backslash \backslash$ Version $=x 86-$ Linux-G03RevB . 03 \State $=2-A^{\prime \prime} \backslash H F=-228.3448224 \backslash S 2=0.7$ $70241 \backslash \mathrm{S} 2-1=0 . \backslash \mathrm{S} 2 \mathrm{~A}=0.750119 \backslash \mathrm{RMSD}=5.164 \mathrm{e}-09 \backslash \mathrm{RMSF}=5.469 \mathrm{e}-06 \backslash \mathrm{Dipole}=-0.262$ $2625,0.8707635,0 . \backslash \mathrm{PG}=\mathrm{CS}[\mathrm{SG}(\mathrm{C} 2 \mathrm{H} 3 \mathrm{O} 2)] \backslash \backslash @$

\section{4}

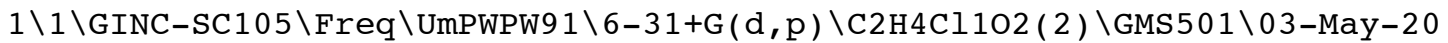
$04 \backslash 0 \backslash \backslash \# P$ MPWPW91/6-31+G(D,P) FREQ=NORAMAN MAXDISK=45875200 GFINPUT IOP $(3 / 76=0572004280) \backslash \backslash E=-689.147853849$ gemdiol chloroacetaldehyde radical mpw1k/6-31+G** C1 symm \\0,2\Cl,-1.6524741837,0.0419529388,-0.25522485 $71 \backslash \mathrm{C},-0.4001401837,-0.1066810612,0.8875941429 \backslash \mathrm{C}, 1.0121808163,-0.029930$ $0612,0.4243941429 \backslash 0,1.3746678163,-1.0983790612,-0.3980188571 \backslash \mathrm{H},-0.6701$ $651837,-0.5625070612,1.8242601429 \backslash \mathrm{H}, 0.7761528163,-1.1211960612,-1.1460$ $678571 \backslash \mathrm{O}, 1.1959648163,1.1860299388,-0.2210078571 \backslash \mathrm{H}, 1.6582308163,-0.121$ $5870612,1.2984971429 \backslash \mathrm{H}, 2.0905378163,1.2105499388,-0.5575828571 \backslash \backslash$ Versio $\mathrm{n}=\mathrm{DEC}-\mathrm{AXP}-\mathrm{OSF} / 1-\mathrm{G} 03 \mathrm{RevB} .05 \backslash \mathrm{State}=2-\mathrm{A} \backslash \mathrm{HF}=-689.1478538 \backslash \mathrm{S} 2=0.756209 \backslash \mathrm{S} 2-1=$ $0 . \backslash \mathrm{S} 2 \mathrm{~A}=0.750027 \backslash \mathrm{RMSD}=7.267 \mathrm{e}-09 \backslash \mathrm{RMSF}=2.022 \mathrm{e}-06 \backslash \mathrm{Dipole}=0.3649097,-0.1689$ $905,0.1831105 \backslash \mathrm{PG}=\mathrm{C} 01 \quad[\mathrm{X}(\mathrm{C} 2 \mathrm{H} 4 \mathrm{C} 1102)] \backslash \backslash @$

\section{5}

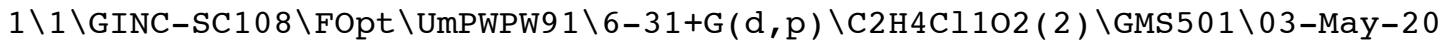
$04 \backslash 0 \backslash \backslash \# P$ MPWPW91/6-31+G(D,P) OPT=(TIGHT, MAXCYCLE $=100)$ MAXDISK=45875200 GFINPUT IOP(3/76=0572004280) \\diol chloroacetaldehyde radical mpw $1 \mathrm{k} / 6$ $-31+\mathrm{G} * * \backslash \backslash 0,2 \backslash \mathrm{C},-0.5192341559,-1.0979116271,-0.6363583613 \backslash \mathrm{C}, 0.51090376$, $-0.0913767875,-0.4564208506 \backslash 0,-1.7979170331,-0.7214682842,-0.680716645$ $4 \backslash \mathrm{H},-1.83342481,0.2216369783,-0.8610657266 \backslash \mathrm{H},-0.3509899781,-2.13112607$ $75,-0.3918121687 \backslash 0,0.1037902307,1.0701892659,-1.0656591245 \backslash \mathrm{H}, 0.6715018$ $399,1.79556933,-0.8104989883 \backslash \mathrm{Cl}, 0.8013299415,0.2879210576,1.3737684877$ $\backslash \mathrm{H}, 1.4933007376,-0.4347755766,-0.763005977 \backslash \backslash$ Version=DEC-AXP-OSF $/ 1-G 03 \mathrm{R}$ evB.05 $\backslash$ state $=2-A \backslash H F=-689.1525026 \backslash S 2=0.757333 \backslash \mathrm{S} 2-1=0 . \backslash \mathrm{S} 2 \mathrm{~A}=0.750038 \backslash \mathrm{RMSD}$ $=6.708 e-09 \backslash \mathrm{RMSF}=5.512 \mathrm{e}-06 \backslash \mathrm{Dipole}=0.50042,0.3097762,-0.6335886 \backslash \mathrm{PG}=\mathrm{C} 01]$ $\mathrm{X}(\mathrm{C} 2 \mathrm{H} 4 \mathrm{Cl} 1 \mathrm{O} 2) \mathrm{]} \backslash \backslash \mathrm{Q}$ 


\section{TS: $1 \rightarrow 7$}

$1 \backslash 1 \backslash G I N C-S C 104 \backslash F T S \backslash U m P W P W 91 \backslash 6-31+G(d, p) \backslash C 4 H 1103(2) \backslash G M S 501 \backslash 31-M a r-2004 \backslash$ $0 \backslash \backslash \# P$ MPWPW91/6-31+G(D,P) OPT=( TS , NOEIGEN , CALCFC, TIGHT $)$ MAXDISK=589824 00 GFINPUT IOP $(3 / 76=0572004280)$ INT=ULTRAFINE $\backslash \backslash \mathrm{TS} 1$ diolethane and Ado= ethanol radical MPW $1 \mathrm{~K} / 6-31+\mathrm{G}(\mathrm{d}, \mathrm{p}) / /$ opt iop $=1$ ultra $\backslash \backslash 0,2 \backslash \mathrm{C}, 2.1819743259$ $,-0.0680870222,0.3365325112 \backslash \mathrm{C}, 1.3723225295,-1.3182438536,0.1640968984 \backslash$ $0,1.9156221041,0.9013861516,-0.6386844445 \backslash \mathrm{H}, 2.0377621138,0.3380445774$, $1.3447110383 \backslash \mathrm{H}, 3.2462641763,-0.2970075712,0.2485996227 \backslash \mathrm{H}, 0.9923397701$, $1.1581382989,-0.5686251162 \backslash \mathrm{H}, 1.404457538,-1.7394293676,-0.8360810076 \backslash \mathrm{H}$ $, 1.5213334143,-2.0669757943,0.9375385862 \backslash \mathrm{C},-1.4480690616,0.6488709991$, $0.8008204639 \backslash \mathrm{C},-1.2720142472,-0.7735377033,0.3665752624 \backslash 0,-0.875408236$ $2,1.4610397121,-0.210365716 \backslash 0,-1.8431705173,-1.0536741152,-0.855115887$ $1 \backslash \mathrm{H}, 0.0265743022,-1.0048380237,0.2998224604 \backslash \mathrm{H},-1.6293782467,-1.4870358$ $332,1.1028894088 \backslash \mathrm{H},-2.5111334053,0.8840856721,0.9042990062 \backslash \mathrm{H},-0.954958$ $3264,0.8126441193,1.7608128067 \backslash \mathrm{H},-1.5751956524,-0.3721667319,-1.471630$ $2985 \backslash \mathrm{H},-1.1396937684,2.3705121461,-0.0971589415 \backslash \backslash$ Version=DEC-AXP-OSF / 1 -G03RevB.03 $\backslash$ State $=2-A \backslash H F=-384.520995 \backslash S 2=0.762758 \backslash S 2-1=0 . \backslash S 2 A=0.750071 \backslash$ $\mathrm{RMSD}=5.390 \mathrm{e}-09 \backslash \mathrm{RMSF}=3.737 \mathrm{e}-07 \backslash \mathrm{Dipole}=-0.8023853,0.5822103,1.0383473 \backslash \mathrm{PG}$ $=\mathrm{C} 01[\mathrm{X}(\mathrm{C} 4 \mathrm{H} 1103)] \backslash \backslash @$

\section{TS:8 $\rightarrow 9$}

$1 \backslash 1 \backslash G I N C-S C 106 \backslash F T S \backslash U m P W P W 91 \backslash 6-31+G(d, p) \backslash C 4 H 1103(2) \backslash G M S 501 \backslash 31-M a r-2004 \backslash$ $0 \backslash \backslash \# \mathrm{P}$ MPWPW91/6-31+G(D,P) OPT=( TS, NOEIGEN, CALCFC, TIGHT $)$ MAXDISK=524288 $00 \operatorname{IOP}(3 / 76=0572004280)$ INT=ULTRAFINE $\backslash \backslash \mathrm{TS} 1$ gemdiolethane radical and $\mathrm{A}$ do=ethanol ultra MPW $1 \mathrm{~K} / 6-31+\mathrm{G}(\mathrm{d}, \mathrm{p}) / /$ opt re-abstraction iop $=1 \backslash \backslash 0,2 \backslash \mathrm{C},-0$ $.8505012608,1.1881706934,-0.4189012126 \backslash \mathrm{C},-1.4473869165,0.0334385566,0$. $3155900526 \backslash \mathrm{H},-0.9378329974,1.0942916247,-1.4958230904 \backslash \mathrm{H},-1.1705445255$, $2.1570197144,-0.049099372 \backslash 0,-2.8246012368,-0.125287332,0.0681788375 \backslash \mathrm{H}$, $-3.3086867684,0.587680327,0.4793498681 \backslash 0,-0.8441780452,-1.1351920531,-$ $0.1355791211 \backslash \mathrm{H},-1.2938720857,0.1409744387,1.3961159849 \backslash \mathrm{H},-1.3624687447$ $,-1.8796738956,0.1676799086 \backslash \mathrm{C}, 2.2525725914,-0.1116473073,0.4904576437 \backslash$ C, $1.803099142,1.2332369651,-0.0044042058 \backslash 0,2.0109850239,-1.1385139052$, $-0.4265362153 \backslash \mathrm{H}, 3.3313209448,-0.0972742192,0.6585257321 \backslash \mathrm{H}, 1.7898806662$ $,-0.3299612327,1.4591437322 \backslash \mathrm{H}, 1.0602235091,-1.2619375961,-0.4860425872$ $\backslash \mathrm{H}, 1.937382307,2.0447925411,0.7065087146 \backslash \mathrm{H}, 0.4922932973,1.1932242518$, $0.1888925507 \backslash \mathrm{H}, 2.177957126,1.4836169217,-0.9924280166 \backslash \backslash$ Version=DEC $-\mathrm{AXP}$ $-\mathrm{OSF} / 1-\mathrm{G} 03 \mathrm{RevB} .03 \backslash \mathrm{State}=2-\mathrm{A} \backslash \mathrm{HF}=-384.5278274 \backslash \mathrm{S} 2=0.762907 \backslash \mathrm{S} 2-1=0 . \backslash \mathrm{S} 2 \mathrm{~A}=0$. $750077 \backslash \mathrm{RMSD}=4.310 \mathrm{e}-09 \backslash \mathrm{RMSF}=6.993 e-08 \backslash \mathrm{Dipole}=-0.902268,0.9268297,1.0334$ $6 \backslash \mathrm{PG}=\mathrm{C} 01 \quad[\mathrm{X}(\mathrm{C} 4 \mathrm{H} 1103)] \backslash \backslash @$

\section{TS: $10 \rightarrow 12$}

$1 \backslash 1 \backslash G I N C-S C 114 \backslash F T S \backslash U m P W P W 91 \backslash 6-31+G(d, p) \backslash C 4 H 1104(2) \backslash G M S 501 \backslash 31-M a r-2004 \backslash$ $0 \backslash \backslash \# \mathrm{P}$ MPWPW91/6-31+G(D,P) OPT=(TS, NOEIGEN , CALCFC, TIGHT) MAXDISK=524288 $00 \operatorname{IOP}(3 / 76=0572004280)$ INT=ULTRAFINE $\backslash \backslash \mathrm{TS} 1 \mathrm{a}$ triolethane and Ado=ethano 1 radical MPW1K/6-31+G**// opt iop=1 ultra \\0,2\0,-0.4816972265,1.2543 $318662,1.0055590829 \backslash \mathrm{C},-0.6202664638,1.004889652,-0.3451674405 \backslash \mathrm{C},-1.479$ $585781,-0.2022826452,-0.6234708639 \backslash \mathrm{H},-0.993742598,1.8882995714,-0.8549$ $861505 \backslash \mathrm{H}, 0.6166268574,0.8153183909,-0.8142469219 \backslash 0,-2.6598743473,-0.14$ $24749966,0.0977302013 \backslash 0,-0.7552015423,-1.3640231635,-0.2756978767 \backslash \mathrm{H},-1$ $.7548758468,-0.2488580089,-1.6764727357 \backslash \mathrm{H}, 0.2541527706,0.7192446739,1$. $3360901742 \backslash \mathrm{H},-2.4434615914,0.2300077658,0.9570480822 \backslash \mathrm{H},-1.3783641302,-$ $2.0869976455,-0.215697825 \backslash \mathrm{C}, 2.3703231978,-0.4688593934,-0.099023456 \backslash \mathrm{C}$, $1.9699670405,0.7553787215,-0.8739206839 \backslash 0,1.5586239183,-0.6721588664,1$ $.043558582 \backslash \mathrm{H}, 2.3324161654,-1.3519449308,-0.737299549 \backslash \mathrm{H}, 3.3947520909,-0$ $.3751112579,0.2626343963 \backslash \mathrm{H}, 0.7871792506,-1.1706057067,0.7517661424 \backslash \mathrm{H}, 2$ $.2273226018,1.697466062,-0.3987636284 \backslash \mathrm{H}, 2.2205600525,0.7430243588,-1.9$ $297772348 \backslash \backslash$ Version=DEC-AXP-OSF /1-G03RevB.03\State=2-A \HF=-459.7313672\ 
$\mathrm{S} 2=0.762932 \backslash \mathrm{S} 2-1=0 . \backslash \mathrm{S} 2 \mathrm{~A}=0.750073 \backslash \mathrm{RMSD}=4.905 \mathrm{e}-09 \backslash \mathrm{RMSF}=3.935 \mathrm{e}-07 \backslash \mathrm{Dipole}=$ $0.1837927,-0.4332175,-0.8194869 \backslash P G=C 01 \quad[X(C 4 \mathrm{H} 1104)] \backslash \backslash @$

\section{TS: $13 \rightarrow 3$}

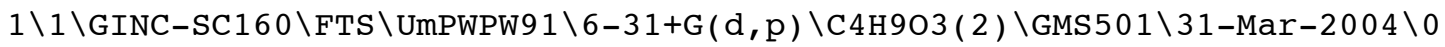
$\backslash \backslash \# P$ MPWPW91/6-31+G(D,P) OPT=(TS, NOEIGEN, CALCFC, TIGHT) MAXDISK $=4849664$ 0 IOP $(3 / 76=0572004280)$ INT=ULTRAFINE $\backslash \backslash T S 4 \mathrm{~b}$ glycolradical and Ado=ethan ol iop=1 MPW1K/6-31+G(d,p)//opt re-abstraction ultra \\0,2\C,-1.5613073 $557,-0.3890270267,-0.7032480621 \backslash \mathrm{C},-1.095886913,0.9288935682,-0.2383965$ $239 \backslash 0,-0.9175140885,1.1183691818,1.1018189577 \backslash \mathrm{H},-0.2178647731,0.524891$ $8508,1.4033538427 \backslash 0,-1.349581213,-1.4323995493,-0.1228595625 \backslash \mathrm{H},-1.6472$ $842009,1.7558327163,-0.6764098411 \backslash \mathrm{H},-2.0501270076,-0.3942866965,-1.690$ $0828944 \backslash C, 1.8442284051,-0.4597494756,-0.241560744 \backslash C, 1.5397915103,0.915$ $9121563,-0.7469004663 \backslash \mathrm{O}, 1.1455734213,-0.7635029475,0.9475282747 \backslash \mathrm{H}, 2.91$ $12630482,-0.5360455371,-0.0166037929 \backslash \mathrm{H}, 1.6307762337,-1.2047091232,-1.0$ $092564131 \backslash \mathrm{H}, 0.3969576442,-1.3326224958,0.7389703385 \backslash \mathrm{H}, 1.8237488749,1.1$ $312380566,-1.770460008 \backslash \mathrm{H}, 0.0505800894,0.9715161495,-0.7604522893 \backslash \mathrm{H}, 1.7$ $131712527,1.7282762654,-0.0503255239 \backslash \backslash$ Version=DEC-AXP-OSF $/ 1-G 03$ RevB 03 $\backslash$ State $=2-\mathrm{A} \backslash \mathrm{HF}=-383.3032401 \backslash \mathrm{S} 2=0.76634 \backslash \mathrm{S} 2-1=0 . \backslash \mathrm{S} 2 \mathrm{~A}=0.750149 \backslash \mathrm{RMSD}=4.475 \mathrm{e}$ $-09 \backslash \mathrm{RMSF}=8.387 \mathrm{e}-07 \backslash \mathrm{Dipole}=0.4458511,0.4443006,-1.3913155 \backslash \mathrm{PG}=\mathrm{C} 01 \quad[\mathrm{X}(\mathrm{C} 4 \mathrm{H}$ $903)] \backslash \backslash @$

\section{TS: $11 \rightarrow 14$}

$1 \backslash 1 \backslash$ GINC-SC160 \FTS \UMPWPW9 $1 \backslash 6-31+\mathrm{G}(\mathrm{d}, \mathrm{p}) \backslash \mathrm{C} 4 \mathrm{H} 10 \mathrm{Cl} 1 \mathrm{O} 3(2) \backslash \mathrm{GMS} 501 \backslash 03-\mathrm{May}-20$ $04 \backslash 0 \backslash \backslash \# P$ MPWPW91/6-31+G(D,P) OPT=(TIGHT, CALCFC, TS, NOEIGENTEST $)$ MAXDISK $=58982400$ GFINPUT IOP $(3 / 76=0572004280) \backslash \backslash$ lowest conformer gemdiol chlor oacetaldehyde mpw1k/6-31+G** C1 symm TS Ado=eth $\backslash \backslash 0,2 \backslash \mathrm{Cl},-1.1560462965$, $1.5925077575,-0.5365701587 \backslash \mathrm{C},-0.7122888682,0.4822254263,0.7461855466 \backslash \mathrm{C}$ $,-1.1105719088,-0.9411063206,0.4729122948 \backslash 0,-2.4739542132,-1.108814309$ $3,0.2923511383 \backslash \mathrm{H},-1.0984539578,0.8440186073,1.6915765015 \backslash \mathrm{H},-2.76940647$ $83,-0.4867948958,-0.3755463774 \backslash 0,-0.4018279669,-1.3928450571,-0.646101$ $8276 \backslash \mathrm{H},-0.8579866617,-1.5353869945,1.352493104 \backslash \mathrm{H},-0.7477958327,-2.2513$ $729924,-0.887826816 \backslash \mathrm{H}, 0.6362415444,0.5508768937,0.8813598617 \backslash \mathrm{C}, 2.59560$ $08739,0.2478778507,-0.254011374 \backslash \mathrm{C}, 1.9453563661,0.7079586608,1.01768081$ $2 \backslash 0,2.4461488069,-1.1249087031,-0.4735935313 \backslash \mathrm{H}, 2.2175291029,0.82960059$ $72,-1.100102306 \backslash \mathrm{H}, 3.6697659278,0.4358597892,-0.1985077292 \backslash \mathrm{H}, 1.51331627$ $62,-1.304316922,-0.6183966236 \backslash \mathrm{H}, 2.189727714,0.1019166631,1.8854978403 \backslash$ $\mathrm{H}, 2.0283376146,1.7737782293,1.2132953298 \backslash \backslash$ Version=DEC-AXP-OSF / 1-G03Rev B. $05 \backslash$ State $=2-\mathrm{A} \backslash \mathrm{HF}=-844.1371047 \backslash \mathrm{S} 2=0.762564 \backslash \mathrm{S} 2-1=0 . \backslash \mathrm{S} 2 \mathrm{~A}=0.750075 \backslash \mathrm{RMSD}=5$ $.902 \mathrm{e}-09 \backslash \mathrm{RMSF}=2.864 \mathrm{e}-06 \backslash \mathrm{Dipole}=-0.5107503,0.1465992,0.4472034 \backslash \mathrm{PG}=\mathrm{C} 01 \quad[$ $\mathrm{X}(\mathrm{C} 4 \mathrm{H} 10 \mathrm{Cl} 1 \mathrm{O} 3)] \backslash \backslash @$

\section{$13 \cdots$ formate}

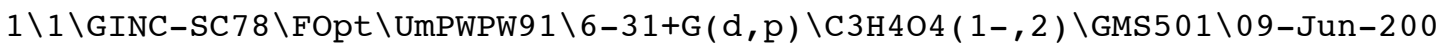
$4 \backslash 0 \backslash \backslash \# \mathrm{P}$ MPWPW91/6-31+G(D,P) OPT $=($ CALCFC, TIGHT, MAXCYCLE $=100)$ MAXDISK $=45$ 875200 GFINPUT IOP $(3 / 76=0572004280) \backslash \backslash \mathrm{COOH}$ over glycolrad mpw $1 \mathrm{k} / 6-31+\mathrm{G} *$ $* \backslash \backslash-1,2 \backslash H,-0.5315136153,0.2124540111,-0.5877482299 \backslash 0,0.5224944723,1.15$ $86675135,-0.4579632931 \backslash \mathrm{C}, 1.7060582139,0.9726174031,-0.0517185761 \backslash \mathrm{C}, 2.3$ $309616473,-0.2532017468,0.2975443651 \backslash 0,1.8353431138,-1.3918649168,0.26$ $72559119 \backslash \mathrm{H}, 2.3509671259,1.8563863896,0.048960949 \backslash \mathrm{H}, 3.3804736881,-0.120$ $2286444,0.6262380737 \backslash 0,-2.5361513037,0.2810844159,0.9360413931 \backslash \mathrm{C},-2.35$ $91728127,-0.3854933081,-0.0579113763 \backslash 0,-1.3387550039,-0.4054098812,-0$. $8466239895 \backslash \mathrm{H},-3.1304597179,-1.0919628967,-0.4046174483 \backslash \backslash$ Version=DEC-AX P-OSF $/ 1-G 03 R e v B .05 \backslash$ State $=2-A \backslash H F=-417.5248765 \backslash \mathrm{S} 2=0.764626 \backslash \mathrm{S} 2-1=0 . \backslash \mathrm{S} 2 \mathrm{~A}=0$ $.750077 \backslash \mathrm{RMSD}=5.554 \mathrm{e}-09 \backslash \mathrm{RMSF}=1.474 \mathrm{e}-06 \backslash \mathrm{Dipole}=-0.1259863,0.2362102,-0.3$ $544944 \backslash \mathrm{PG}=\mathrm{C} 01 \quad[\mathrm{X}(\mathrm{C} 3 \mathrm{H} 4 \mathrm{O} 4)] \backslash \backslash @$ 


\section{$5 \cdot \cdots$ formate}

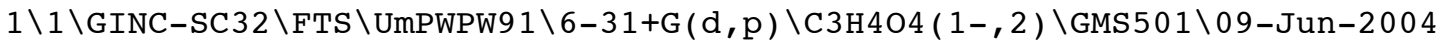
$\backslash 0 \backslash \backslash \# P$ MPWPW91/6-31+G(D,P) OPT=(CALCFC , TS , NOEIGEN , TIGHT, MAXCYCLE $=100)$ MAXDISK $=45875200$ GFINPUT IOP $(3 / 76=0572004280) \backslash \backslash \mathrm{Cs} \mathrm{COOH}$ orthogonal over semidione mpw $1 \mathrm{k} / 6-31+\mathrm{G} * * \backslash \backslash-1,2 \backslash \mathrm{H},-0.3536202024,0.5479485297,0 . \backslash 0,-0.3$ $885744585,-0.9658216361,1.4059589536 \backslash 0,-0.3885744585,-0.9658216361,-1$. $4059589536 \backslash \mathrm{C},-0.3797844898,-2.0066126039,0.7091388652 \backslash \mathrm{C},-0.3797844898$, $-2.0066126039,-0.7091388652 \backslash \mathrm{H},-0.3708862586,-2.9984705858,1.1940031452$ $\backslash \mathrm{H},-0.3708862586,-2.9984705858,-1.1940031452 \backslash \mathrm{O},-0.5569100881,1.5157068$ $188,0 . \backslash \mathrm{C}, 0.4890238841,2.285329225,0 . \backslash \mathrm{O}, 1.652378413,1.9757129497,0 . \backslash \mathrm{H}, 0$ $.1721080295,3.3381565686,0$. \\Version=DEC-AXP-OSF/1-G03RevB .05 State=2$A^{\prime} \backslash \mathrm{HF}=-417.5172814 \backslash \mathrm{S} 2=0.764025 \backslash \mathrm{S} 2-1=0 . \backslash \mathrm{S} 2 \mathrm{~A}=0.750071 \backslash \mathrm{RMSD}=7.168 \mathrm{e}-09 \backslash \mathrm{RMS}$ $\mathrm{F}=5.447 \mathrm{e}-07 \backslash \mathrm{Dipole}=-0.2434091,0.5854995,0 . \backslash \mathrm{PG}=\mathrm{CS}[\mathrm{SG}(\mathrm{C} 1 \mathrm{H} 2 \mathrm{O} 2), \mathrm{X}(\mathrm{C} 2 \mathrm{H} 2 \mathrm{O} 2)$ ] $\backslash \backslash Q$

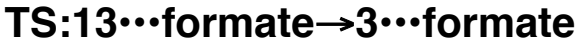

$1 \backslash 1 \backslash G I N C-S C 63 \backslash F T S \backslash U m P W P W 91 \backslash 6-31+G(d, p) \backslash C 5 H 1005(1-, 2) \backslash G M S 501 \backslash 13-J u 1-200$ $4 \backslash 0 \backslash \backslash \# P$ MPWPW91/6-31+G(D,P) OPT $=($ TIGHT, MAXCYCLE $=100, \mathrm{TS}, \mathrm{CALCFC}, \mathrm{NOEIGENT}$ EST) MAXDISK=58982400 GFINPUT IOP $(3 / 76=0572004280) \backslash \backslash \mathrm{TS} 3$ glycolrad form

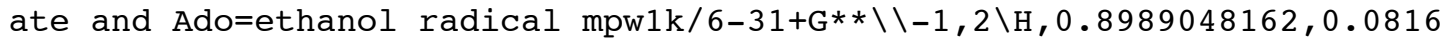
$08002,-1.0072560298 \backslash 0,-0.0494828365,0.4552293018,-0.9846410255 \backslash \mathrm{C},-0.27$ $87879098,0.9218621589,0.2710385887 \backslash C,-1.1703165105,2.0799784222,0.4032$ $813741 \backslash \mathrm{O},-1.8254885541,2.6113181013,-0.4617845497 \backslash \mathrm{H}, 0.6301700226,1.015$ $9584638,0.8751598601 \backslash \mathrm{H},-1.2502040672,2.4294659542,1.4521523033 \backslash 0,2.746$ $2582856,0.3400871671,1.0674409618 \backslash \mathrm{C}, 3.0199613839,-0.277290425,0.033999$ $6481 \backslash 0,2.2904255307,-0.4918987329,-0.9690845983 \backslash \mathrm{H}, 4.0395404987,-0.7119$ $444616,-0.0419439819 \backslash \mathrm{C},-1.2584803751,-2.1490678734,0.1948698509 \backslash \mathrm{C},-1.5$ $423739525,-1.2547202741,1.3605406778 \backslash 0,-1.8218242919,-1.6743469384,-0$. $9958604612 \backslash \mathrm{H},-1.6864693957,-3.142351367,0.3672964268 \backslash \mathrm{H},-0.1775886242,-$ $2.2828346557,0.0910267426 \backslash \mathrm{H},-1.3073650168,-0.8926774467,-1.2485262113 \backslash$ $\mathrm{H},-1.0003593581,-1.45120102,2.2791628433 \backslash \mathrm{H},-0.909262102,0.0593648166,0$ $.9134807319 \backslash \mathrm{H},-2.5764876606,-0.9530715293,1.4885038616 \backslash \backslash$ Version=DEC $-\mathrm{AX}$ P-OSF / 1-G03RevB.05 \State $=2-A \backslash H F=-572.4924441 \backslash S 2=0.765817 \backslash \mathrm{S} 2-1=0 . \backslash \mathrm{S} 2 \mathrm{~A}=0$ $.750144 \backslash \mathrm{RMSD}=4.846 \mathrm{e}-09 \backslash \mathrm{RMSF}=3.121 \mathrm{e}-06 \backslash \mathrm{Dipole}=-0.9602807,-0.6716346,1.3$ $73866 \backslash \mathrm{PG}=\mathrm{C} 01 \quad[\mathrm{X}(\mathrm{C} 5 \mathrm{H} 10 \mathrm{O} 5)] \backslash \backslash @$ 
TABLE S2: G3(MP2)-RAD//MPW1K/6-31+G(d,p) Total Energies (Hartrees) at O K

\begin{tabular}{|c|c|}
\hline Species & \\
\hline 1 & -229.93181 \\
\hline 2 & -269.17310 \\
\hline 3 & -228.73902 \\
\hline 4 & -612.75320 \\
\hline 5 & -228.09675 \\
\hline 6 & -153.59921 \\
\hline 7 & -229.28098 \\
\hline 8 & -229.28642 \\
\hline 9 & -229.95010 \\
\hline 10 & -305.09265 \\
\hline 11 & -689.10850 \\
\hline 12 & -304.43931 \\
\hline 13 & -228.11124 \\
\hline 14 & -688.44920 \\
\hline 15 & -688.45142 \\
\hline TS: $1 \rightarrow 7$ & -384.04694 \\
\hline TS:8 $\rightarrow 9$ & -384.05575 \\
\hline TS: $10 \rightarrow 12$ & -459.20816 \\
\hline TS: $13 \rightarrow 3$ & -382.85777 \\
\hline TS: $11 \rightarrow 14$ & -843.22327 \\
\hline 13॰•formate & -417.14187 \\
\hline $5 \cdot \bullet \bullet$ formate & -417.13651 \\
\hline TS:13॰••formate $\rightarrow$ 3॰••formate & -571.89738 \\
\hline
\end{tabular}

